\section{Thyroxin-Index, freier}

W. Hubl

Ehem. Institut für Klinische Chemie und Labormedizin, Krankenhaus Dresden-Friedrichstadt, Dresden, Deutschland

Synonym(e) Freier T4-Index; FT4I; FTI

Englischer Begriff free thyroxine index; FTI

Definition Der Freie Thyroxin-Index stellt ein Maß für das Verhältnis von freien, nicht proteingebundenen zu proteingebundenem Thyroxin dar. Hiermit gelingt es, eine Abschätzung des freien, biologisch aktiven Thyroxins vorzunehmen, um im Zusammenhang mit der Bestimmung des GesamtThyroxins die Funktion der Schilddrüse besser zu beurteilen.

Beschreibung Bei den traditionellen Bestimmungen der Gesamt-Schilddrüsenhormone, die aufgrund der Abhängigkeit von der Konzentration der Bindungsproteine sehr störanfällig sind, wurde der Versuch unternommen, Kriterien für eine Einschätzung der Konzentration der freien Hormone zu erlangen. Eine Möglichkeit war der Freie Thyroxin-Index, der mit der Konzentration des freien Thyroxins korreliert.

Dieser Index wird aus dem Quotienten der GesamtThyroxinbestimmung sowie einem Parameter zur Abschätzung der Proteinbindung, z. B. durch direkte Bestimmung von $>$ Thyroxin-bindendes Globulin oder einem indirekten Bindungstest, wie dem T3- oder T4-Uptake-Test ( $\triangleright$ Thyroxinbindungskapazität), berechnet.

Durch die Einführung des Freien Thyroxin-Index wurde die Schilddrüsen-Funktionsdiagnostik deutlich verbessert mit einer Steigerung der diagnostischen Sensitivität für Schilddrüsenerkrankungen.

Analytik: Zur Bestimmung mit der Indexmethode im Blutserum werden Radioimmunoassay ( Radioimmunoassay) bzw. Enzymimmunoassays ( $\triangleright$ Enzymimmunoassay) eingesetzt.

\section{Referenzbereiche:}

\begin{tabular}{l|l|}
\hline Frauen: & $73-154 \mathrm{nmol} / \mathrm{L}$ \\
\hline Männer: & $72-147 \mathrm{nmol} / \mathrm{L}$ \\
\hline Kinder und Jugendliche: & \\
\hline $0-6$ Tage: & $65-268 \mathrm{nmol} / \mathrm{L}$ \\
\hline$>6$ Tage -3 Monate: & $71-232 \mathrm{nmol} / \mathrm{L}$ \\
\hline$>3-12$ Monate: & $73-216 \mathrm{nmol} / \mathrm{L}$ \\
\hline$>1-6$ Jahre: & $77-179 \mathrm{nmol} / \mathrm{L}$ \\
\hline $11-20$ Jahre: & $76-170 \mathrm{nmol} / \mathrm{L}$ \\
\hline
\end{tabular}

Nach Einführung der direkten Bestimmungen für die freien Schilddrüsenhormone hat der Freie Thyroxin-Index heute an Bedeutung verloren.

\section{Literatur}

Bourcigaux N, Lepoutre-Lussey C, Guéchot J (2010) Thyroid function at the third trimester of pregnancy in a Northern French population. Ann Endocrinol (Paris) 71:519-524

Hörmann R (2007) Schilddrüsenkrankheiten - Leitfaden für Praxis und Klinik. ABW Wissenschaftsverlag, S 50/93

Vogt W (1995) Schilddrüsenhormone. In: Greiling H, Gressner AM (Hrsg) Lehrbuch der Klinischen Chemie und Pathobiochemie, 3. Aufl. Schattauer, Stuttgart/New York, S 1011 